FERMILAB-TM-2421-DO

October 2008

\title{
Accelerator/Experiment Operations - FY 2008
}

\author{
S. Brice, M. Buehler, M. Casarsa, R. Coleman, D. Denisov, G. Ginther, \\ S. Grinstein, A. Habig, S. Holmes, J. Hylen, W. Kissel, W. Lee, T. Nakaya, \\ R. Plunkett, E. Ramberg, P. Schlabach, R. Van de Water, M. O. Wascko, D. Wood
}

Edited by J. A. Appel

This Technical Memorandum (TM) summarizes the Fermilab accelerator and accelerator experiment operations for FY 2008. It is one of a series of annual publications intended to gather information in one place. In this case, the information concerns the FY 2008 Run II at the Tevatron Collider, MINOS using the Main Injector Neutrino Beam (NuMI), the MiniBooNE and SciBooNE experiments running in the Booster Neutrino Beam (BNB), and the Meson Test Beam (MTest) activities in the $120 \mathrm{GeV}$ external Switchyard beam (SY120).

Each section was prepared by the relevant authors, and was somewhat edited for inclusion in this summary.

\section{Accelerator Operations (S. Holmes)}

\section{$\underline{\text { Tevatron Collider }}$}

The accelerators entered FY 2008 nearing the end of the 2007 long shutdown. The shutdown was completed on schedule. All planned work was completed with the exception of replacement of the flexible low-conductivity water (LCW) hoses in the Main Injector. Tevatron Collider operations recommenced on October 28, and continued through the balance of FY 2008. The recovery of the accelerator complex from the shutdown was complicated by the introduction of a new Tevatron helix intended to reduce beam losses during the squeeze. Complications, including an offset in the interaction point at B0, caused the Accelerator Division to retreat to the previous helix within two weeks. The 2008 shutdown, originally scheduled to start in September 2008, was postponed to mid-June, 2009 in order to maximize running time before the cessation of Collider operations planned for October 1, 2010. For FY 2008 (defined as 0000 on October 1, 2007 to 2400 on September 29, 2008) integrated luminosity (average of CDF and DZero) totaled $1789.5 \mathrm{pb}^{-1}, 105 \%$ of the design goal and $136 \%$ of that delivered in FY 2007. Over 48 weeks of scheduled operations, a total of 5032 store hours was delivered, $100 \%$ of the design goal. Total on-line average integrated luminosity for Run II stood at $4.993 \mathrm{fb}^{-1}$ as of 9/29/08.

Highlights for the year include:

- A record initial luminosity (average of CDF and DZero) of $31.8 \times 10^{31}$ on July 5 - a $9 \%$ increase over the record at the end of FY 2007.

- A record weekly delivered luminosity (average of CDF and DZero) of $57.5 \mathrm{pb}^{-1}$ over the period June 30 - July 6 - a 28\% increase over the record at the end of FY 2007. 
- Record integrated luminosity (average of CDF and DZero) delivered in a month (May) of $221.4 \mathrm{pb}^{-1}-\mathrm{a} 33 \%$ increase over the record at the end of FY 2007.

- Improvements to the antiproton stacking rate. The record for antiprotons stacked in an hour was set at $27.0 \times 10^{10} /$ hour - a $17 \%$ improvement over FY 2007. The average sustainable stacking rate rose to $24-25 \times 10^{10}$ /hour.

- Reduction of the time required to transfer antiprotons from the Accumulator to the Recycler. These transfers now routinely take 8 minutes.

- Good reliability, with unscheduled downtime reduced to $16 \%$, as compared to $17 \%$ in FY 2007 and 23\% in FY 2006. This was achieved despite 1.5 weeks of unscheduled downtime due to a superconducting component failure over the Christmas holiday period.

- All these achievements were made in the face of four months of forced furloughs and impending involuntary reductions in force.

The stage was set for these improvements by the successful completion of the Run II Luminosity Upgrade Program at the end of FY 2006. As planned for the balance of Run II, the Collider program is capitalizing on the investments made over FY 2003-2006 to optimize running conditions and maximize integrated luminosity.

\section{FY2008 Luminosity}

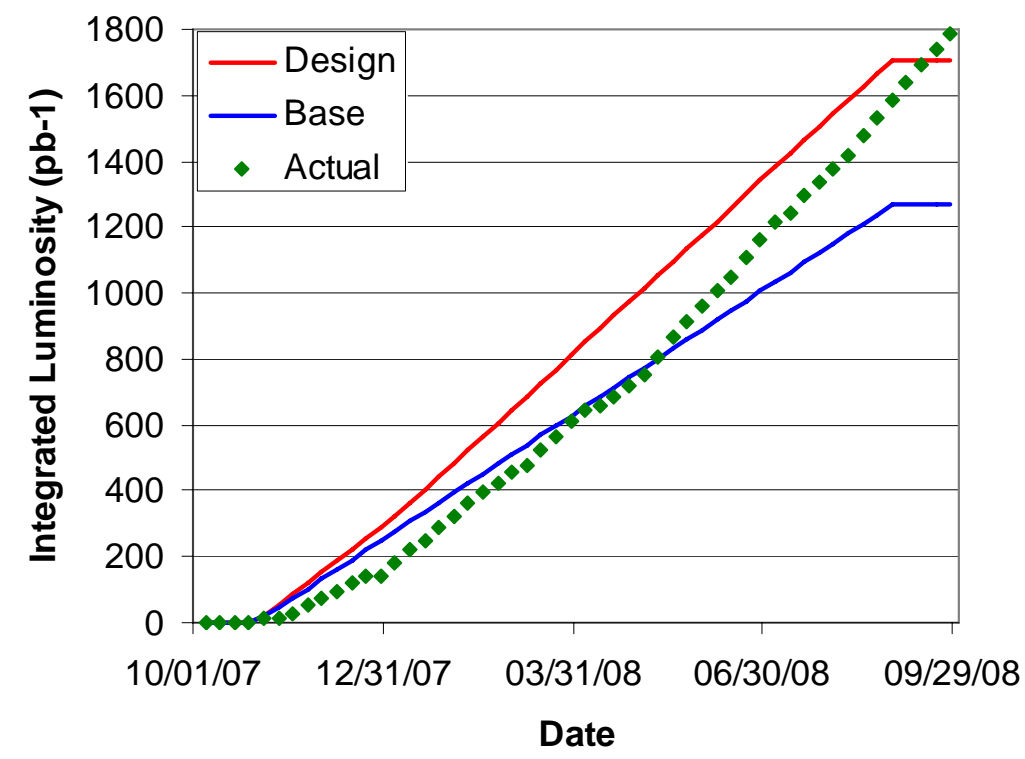

Figure 1. FY 2008 integrated luminosity, planned and delivered (average of CDF and DZero).

\section{Commentary on FY 2008 Collider Performance}

The twenty-store running average of Collider initial luminosity reached an all time high of $28.9 \times 10^{31} \mathrm{~cm}^{-2} \mathrm{~s}^{-1}$ in July. This represents a $20 \%$ improvement over FY 2007, and was $90 \%$ of 
the design goal of $32 \times 10^{31}$. Improved performance over the course of the year was derived from a number of sources, some anticipated and some not. The most significant factors were related to the number of antiprotons brought into collision - up by roughly $20 \%$ over the last year. Primary contributors are:

- The stacking rate was improved to roughly $24-25 \times 10^{10}$ /hour (15-20\% improvement over FY 2007). The primary drivers were improvements to the stack-tail and Debuncher cooling systems and a significant increase in the lithium lens gradient.

- Interruptions to antiproton stacking during transfers between the Accumulator and Recycler were reduced significantly over the year, and now average about 8 minutes with $90 \%$ transfer efficiency.

Reliability in FY 2008 was good. For the year an average of 105 hours of luminosity per scheduled week of operations was reached - a 4\% decrease from FY 2007, but still at the design goal. This was achieved despite a major cryogenic component failure that required repair over the Christmas holiday, interrupting operations for a total of 1.5 weeks, out of 48 weeks scheduled. The antiproton stacking rate is the primary key to performance in the Tevatron. The design luminosity goal for 2008 was based on a stacking rate of $30 \times 10^{10} /$ hour. As noted above, $24-25 \times 10^{10}$ /hour was achieved. A number of improvements to the antiproton cooling systems were implemented. The Collider routinely operates with the new-style lithium lens at $760 \mathrm{~T} / \mathrm{m}$. Good transfer efficiencies and emittance control within the Recycler have allowed coming to within a few percent of the luminosity design goal, despite a 15-20\% shortfall in the stacking rate. Significant modifications to the accelerator complex are complete, and the emphasis over the last two years of Collider operations will be on reliability of operations to maximize integrated luminosity in the Tevatron.

Antiproton availability remains the most important variable determining the luminosity of any particular store. Current experience is that luminosities in the range $2.7-3.2 \times 10^{32}$ are sustainable with a $25-26 \times 10^{10}$ /hour stacking rate as long as stores remain in the Tevatron for 15 18 hours. For the balance of Run II, the luminosity is expected to be roughly proportional to the stacking rate.

\section{Status Relative to the FY 2008 Plan}

Table 1 shows planned and actual performance for the year.

Table 1. 2008 Tevatron Collider planned and actual performance.

\begin{tabular}{|l|l|l|l|}
\hline & Base Profile & Design Profile & Actual \\
\hline Median initial luminosity $\left(\mathrm{cm}^{-2} \mathrm{~s}^{-1}\right)$ & $1.1 \times 10^{32}$ & $3.2 \times 10^{32}$ & $2.8 \times 10^{32}$ \\
\hline Protons/bunch & $250 \times 10^{9}$ & $260 \times 10^{9}$ & $253 \times 10^{9}$ \\
\hline Pbars/bunch & $38 \times 10^{9}$ & $100 \times 10^{9}$ & $78 \times 10^{9}$ \\
\hline Effective emittance $(\pi \mathrm{mm}-\mathrm{mr})$ & 16 & 15 & 15 \\
\hline Beta at the IP $(\mathrm{cm})$ & 30 & 30 & 28 \\
\hline Hourglass factor & 0.65 & 0.65 & 0.67 \\
\hline Average stacking rate $\left(10^{10}\right.$ /hour $)$ & 16 & 30 & 23 \\
\hline
\end{tabular}




\begin{tabular}{|l|l|l|l|}
\hline FY 2008 integrated luminosity $\left(\mathrm{pb}^{-1}\right)$ & 1266 & 1708 & 1790 \\
\hline FY 2008 integrated store time (hours) & 4848 & 5040 & 5032 \\
\hline FY 2008 scheduled uptime (hours) & & & 7714 \\
\hline FY 2008 unscheduled downtime (hours) & & & 1198 \\
\hline $\begin{array}{l}\text { FY 2008 unscheduled downtime as percent } \\
\text { of scheduled uptime }\end{array}$ & & & $16 \%$ \\
\hline
\end{tabular}

"Base" and "Design" correspond to end-of-year goals. Except for the last six rows, "Actual" corresponds to simultaneous performance on the median store of the last four weeks of operations.

\section{FY 2008 Neutrino Operations}

NuMI operations achieved roughly $75 \%$ of the design goal for the year. A total of $2.0 \times 10^{20}$ protons were delivered to the NuMI target in FY2008 - modestly higher than the $1.9 \times 10^{20}$ delivered protons in FY 2007. The primary shortfall relative to the design goal arose from the reliability - nearly 12 weeks of scheduled operations were lost over the year due to: 1) delay to the startup due to installation, and subsequent repairs of, target hall dehumidification (for tritium mitigation); 2) repair of a horn 2 water leak; 3) replacement of a failed beamline magnet; and 4) replacement of failed horn 1. However, protons delivered on target were sustained at higher levels than in FY 2007 due to the implementation of multi-batch slip stacking in the Main Injector in December. This mode of operation supported roughly $260 \mathrm{~kW}$ of beam power on the NuMI target coincident with antiproton stacking, and in excess of $300 \mathrm{~kW}$ during periods of dedicated NuMI operations (with a record of $315 \mathrm{~kW}$ on December 27). Since the beginning of NuMI operations in 2005, the total protons delivered now stands at $5.6 \times 10^{20}$.

Efforts continued on the mitigation of tritium production and releases from the NuMI facility. There were no measurable concentrations of tritium in any of the streams flowing off the Fermilab site in FY 2008. A target chase dehumidification system was installed in the FY 2007 shutdown and is now operational. This system has led to an additional factor of two reduction in tritium pumped from NuMI into the surface holding tank. Tritium levels in all surface ponds are now below detectable levels with the exception of Kidney Pond which is $\sim 1.5 \mathrm{pC} / \mathrm{ml}$ - slightly above detectable and well isolated from outflowing streams.

MiniBooNE operations benefited greatly from interruptions to NuMI operations and hence finished the year well above their design curve $-2.3 \times 10^{20}$ delivered as compared to a design curve of $1.3 \times 10^{20}$. The SciBooNE experiment received its quota of protons and ceased data-taking on August 18. MiniBooNE continues to run in anti-neutrino mode.

\section{Shutdown Activities}

There was no major accelerator shutdown in FY 2008. A long-shutdown had begun near the end of FY 2007, and was completed early in FY 2008. The work done during that shutdown is listed in Table 2. A ten-week shutdown originally scheduled for the end of FY 2008 has been postponed to mid-June 2009. The postponement was undertaken in consideration of the potential for a continuation of the staff furloughs implemented earlier in the year in response to budget shortfalls, and in order to position the Laboratory such that only one long shutdown would be required over the balance of Run II, assuming operations continue through FY 2010. 
Table 2. Status of major shutdown activities.

\begin{tabular}{|c|c|c|}
\hline Machine/Activity & Goal & Status \\
\hline \multicolumn{3}{|l|}{ Linac } \\
\hline LLRF upgrade & $\begin{array}{l}\text { Improved flexibility and reliability. } \\
\text { A Proton Plan activity. }\end{array}$ & $\begin{array}{l}\text { All supporting hardware was installed, but } \\
\text { difficulties with the processor board will } \\
\text { delay implementation until after the } \\
\text { shutdown. }\end{array}$ \\
\hline MTA $400 \mathrm{MeV}$ beamline & $\begin{array}{l}\text { Installation of components to allow } \\
\text { delivery of } 400 \mathrm{MeV} \text { beam to the } \\
\text { MuCool Test Area. }\end{array}$ & $\begin{array}{l}\text { Complete. All components scheduled for } \\
\text { installation during the shutdown were in. } \\
\text { Delivery of beam will require the further } \\
\text { installation of a "C-magnet", to be available } \\
\text { in late fall. }\end{array}$ \\
\hline \multicolumn{3}{|l|}{ Booster } \\
\hline New Booster correctors & $\begin{array}{l}\text { Loss reduction during acceleration. } \\
\text { A Proton Plan activity. }\end{array}$ & $\begin{array}{l}\text { Complete. Twelve correctors were installed } \\
\text { and will be operational during the coming } \\
\text { year. The remaining } 36 \text { will be installed in } \\
\text { the } 2009 \text { shutdown. }\end{array}$ \\
\hline \multicolumn{3}{|l|}{ Antiproton Source } \\
\hline $\begin{array}{l}\text { Replace leaking large quadrupole in the } \\
\text { Accumulator }\end{array}$ & Reliability risk reduction & Complete \\
\hline Replace lithium lens & Increased antiproton yield & Complete \\
\hline \multicolumn{3}{|l|}{ Main Injector } \\
\hline Install MI-30 collimation system & $\begin{array}{l}\text { Increased intensity to NuMI: } \\
\text { control of losses to allow } \\
\text { implementation of multi-batch slip } \\
\text { stacking. A Proton Plan activity. }\end{array}$ & Complete \\
\hline \multicolumn{3}{|l|}{ Recycler } \\
\hline Install new DCCT & Improved diagnostics & Complete \\
\hline $\begin{array}{l}\text { Find/repair leaks in the antiproton } \\
\text { extraction line }\end{array}$ & Improved reliability & $\begin{array}{l}\text { Complete. Two leaks which had been } \\
\text { causing operational problems were } \\
\text { identified and repaired. }\end{array}$ \\
\hline \multicolumn{3}{|l|}{ Tevatron } \\
\hline Unroll Tevatron dipoles & Global coupling reduction & $\begin{array}{l}\text { Complete. All magnets were aligned to } \\
\text { within } 1 \mathrm{mr} \text { roll. ( } 55 \text { magnets unrolled) }\end{array}$ \\
\hline Repair vacuum & Improved reliability & $\begin{array}{l}\text { Six (of 24) houses were warmed up. } \\
\text { Multiple leaks were identified and repaired. } \\
\text { One dipole was replaced in A1. Two } \\
\text { dipoles and one spool were replaced in E1. }\end{array}$ \\
\hline \multicolumn{3}{|l|}{ NuMI } \\
\hline Repair horn 2 water leak & Operability & Complete \\
\hline Fill decay pipe with $\mathrm{He}$ & Safety & Completed in early FY 2008 \\
\hline Add target chase dehumidification & Tritium reduction & Completed in early FY 2008 \\
\hline \multicolumn{3}{|l|}{ Infrastructure Maintenance } \\
\hline Replace $345 \mathrm{KV}$ power poles & Reliability risk reduction & Complete \\
\hline
\end{tabular}




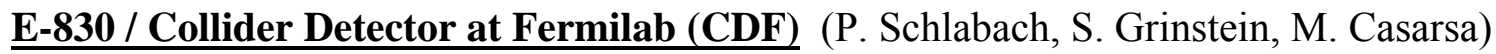

During FY 2008 a total integrated luminosity of $1.8 \mathrm{fb}^{-1}$ was delivered to the CDF detector, of which $1.5 \mathrm{fb}^{-1}$ was recorded to tape. At the end of FY 2008, a total of $5.1 \mathrm{fb}^{-1}$ had been delivered to the experiment in Run II (Figure 2). Overall, the CDF detector has been operating with good live-time and no significant issues, even at the highest instantaneous luminosities. The total data-collection efficiency was $83 \%$, including dead time associated with trigger acceptance, operational inefficiencies (e.g. starting and stopping runs), and downtime from detector problems.

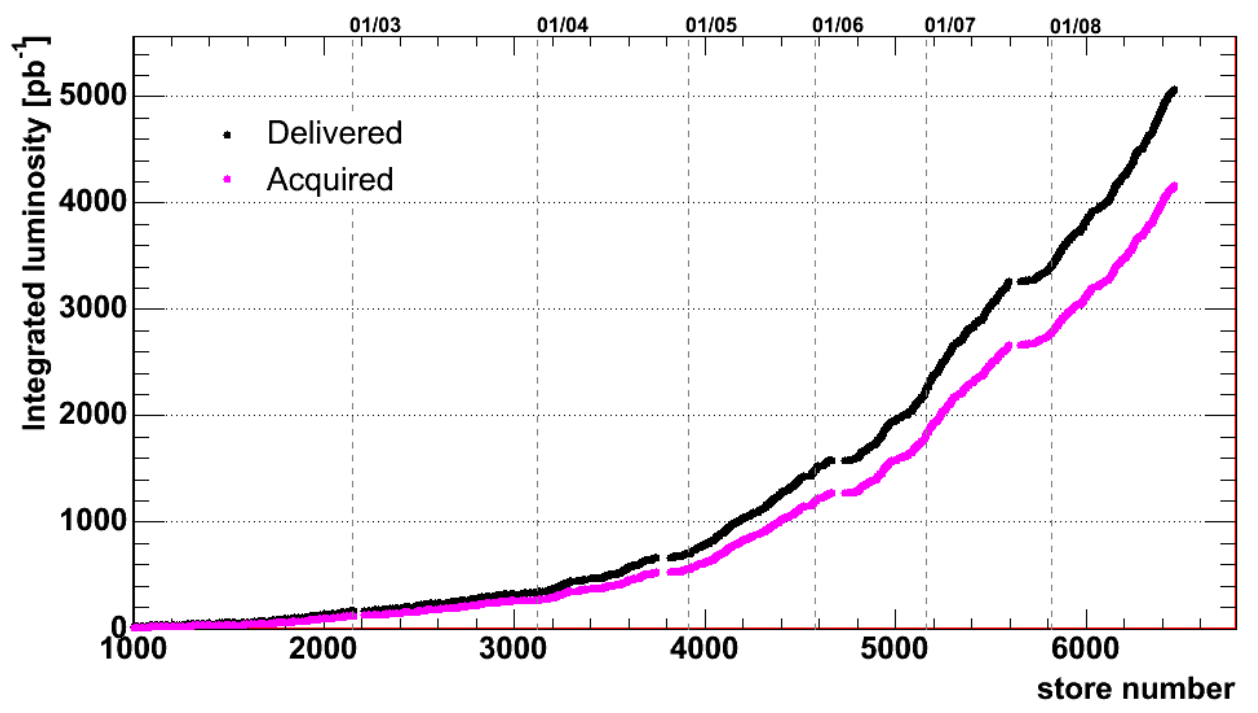

Figure 2. Delivered and recorded luminosity at CDF in fiscal years 2001-2008.

The Tevatron initial instantaneous luminosities were steadily in the range $2.5-3 \times 10^{32}$ $\mathrm{cm}^{-2} \mathrm{~s}^{-1}$ with several stores starting above $3 \times 10^{32} \mathrm{~cm}^{-2} \mathrm{~s}^{-1}$. In order to take advantage of the higher instantaneous luminosities, the CDF trigger underwent further improvements, both in the hardware and in the decision algorithms. The final commissioning of the stereo upgrade of the track trigger at Level 2 was completed, and a new system was set up for the calculation of the missing transverse-energy at Level 1. Those improvements, along with the upgraded Level 2 calorimeter trigger, allowed the implementation of new and more sophisticated trigger selections which allowed a better utilization of the available bandwidth, and significantly increased the purity of the collected data samples.

The track trigger started to take advantage of the stereo layers of the central tracker from March 2007. The stereo track segments are used to confirm that the Level 1 axial track has corresponding segments in the stereo layers, significantly reducing the rate of fake tracks. During the first months of FY 2008, the extension to Level 2 of the stereo trigger upgrade was completely commissioned. The new system exploits the information of the stereo segments and provides three-dimensional track information to the Level 2 decision node. The critical Level 2 high-transverse-momentum muon triggers, used for top, Higgs, and other searches, benefit the most from the upgraded system in the high-luminosity regime. 
The new missing-transverse-energy system (L1MET) provides the Level 1 decision processor with an event missing transverse-energy that has a resolution comparable to the one calculated by the offline event reconstruction. It represents a direct extension and completion of the recently upgraded Level 2 calorimeter trigger, and partly utilizes the same hardware. The energy information from the trigger towers is made available to Level 1 and Level 2 systems at the same time. The L1MET hardware calculates the missing transverse-energy, compares the value to different thresholds, and sends the result back to the Level 1 trigger in time for the Level 1 processor to produce a decision. Currently the upgrade is in production with no operational issues and is performing as expected. Above an instantaneous luminosity of $3 \times 10^{32}$ $\mathrm{cm}^{-2} \mathrm{~s}^{-1}$, it allows a gain of $2 \mathrm{kHz}$ of Level 1 bandwidth and a factor of two reduction in dead time.

The beginning of the fiscal year brought the return of data-taking after a shutdown of approximately two months. The return to routine data-taking took approximately until the end of the calendar year, both from the point of view of delivered luminosity and shift operations. This can be seen in the data-taking efficiency (Figure 3).

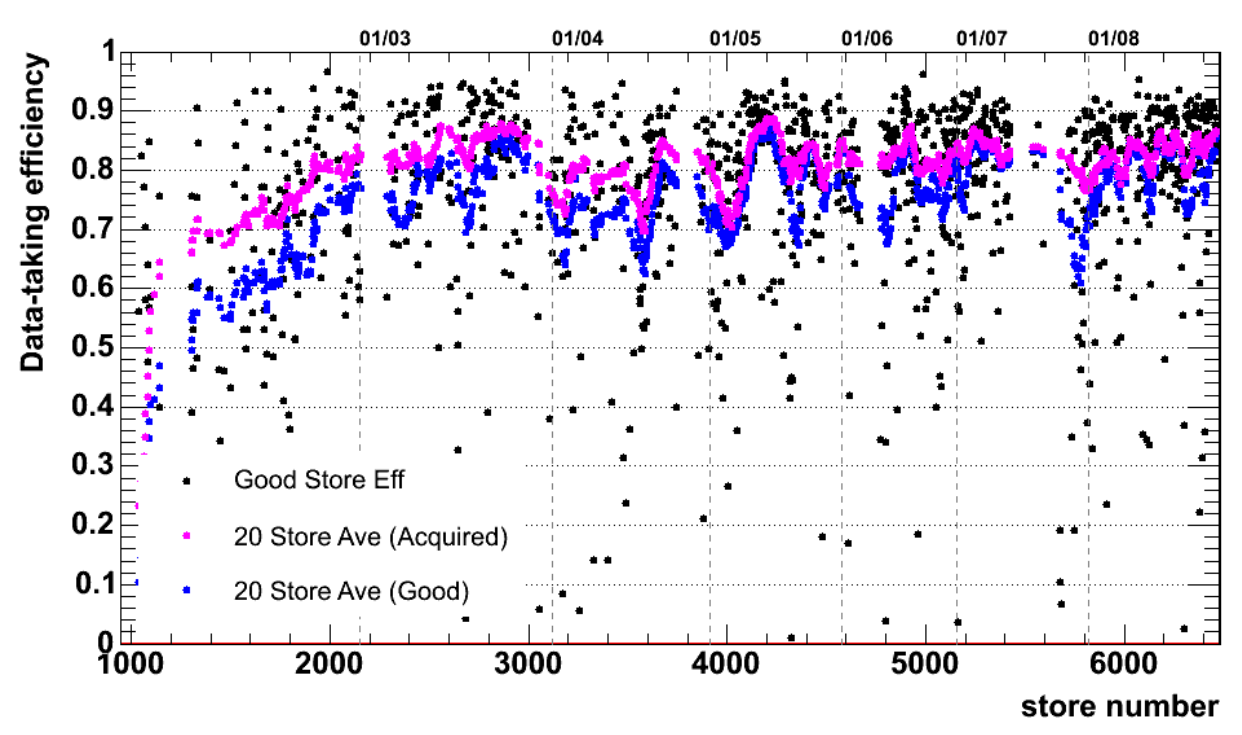

Figure 3. Data-taking efficiency for CDF in fiscal years 2001-2008.

Repair of the L00/ISL silicon tracking system cooling was the primary focus at CDF for the 2007 shutdown. The repairs led to stable operations, and there were no particular issues since the repairs were made. Aging of wires in the Central Outer Tracker (COT) is also not an issue. There were a couple of new problems that arose during the year. The first involved issues with contamination in the cryogenic system for the solenoid. The system has been running without warming up for many years now, leading to a buildup of contaminants. Over the course of the year, contaminants would freeze out at places in the system where there was a restricted aperture, clogging flow. There were a number of different places where this happened. Procedures were developed to break up, blow out or melt the clogs, but each new location cost downtime until the process became routine. CDF also had to open the end plugs a number of times to disable 
glowing wires in the COT. This required about 1.5 shifts of interruption to Tevatron beam. More importantly, each instance required at least several days to localize the wires involved. During these periods, data-taking was significantly disrupted by the localization. After the last of these, the failures were noted to be at a specific location in the cell. In July, the voltages for the wires in this location were reduced to lower the gain. Data-taking has not been interrupted again for this problem. During the one-week shutdown planned for October 2008, CDF will open both end plugs and perform preventive maintenance aimed at mitigation of potential HV problems in the COT.

Another significant activity for the year was planning, and then preparing to replace the emergency generator. Its reliability and the availability of parts made continued operation problematic. The plan is to move to a temporary generator during the short shutdown; remove the old generator; and then install the new one during some future downtime. Other shutdown activities planned in addition to routine maintenance include beam pipe alignment in the hall, installation of new disks for the online database, and a new webserver machine.

There were no major changes in shift operations. The number of offshore institutions participating in remote data-quality monitoring went from one to three as Japan and Korea came online. CDF continued to move some responsibilities from physicist support to technician support. Primary examples were silicon cooling and power supply monitoring. This continues to evolve. CDF also had to shift responsibilities for a few major systems (event builder/Level 3 trigger) as institutions and people gradually move to the LHC. This was accomplished without issue.

$\underline{\text { E-823 / DZero (DZero) }}$ (M. Buehler, D. Denisov, G. Ginther, W. Lee, D. Wood)

The accelerator complex delivered an integrated luminosity of $1.77 \mathrm{fb}^{-1}$ to DZero during FY 2008. DZero recorded $1.61 \mathrm{fb}^{-1}$, corresponding to an operating efficiency of $90.6 \%$ during that time period. Figure 4 illustrates the detector efficiency as a function of time during Run II. The FY 2008 performance represents the best yearly operating efficiency achieved by DZero to date. These achievements were made possible by increases in instantaneous and integrated luminosities delivered by the accelerator complex, and operational improvements in the detector, several of which were introduced during the 2007 long shutdown. 


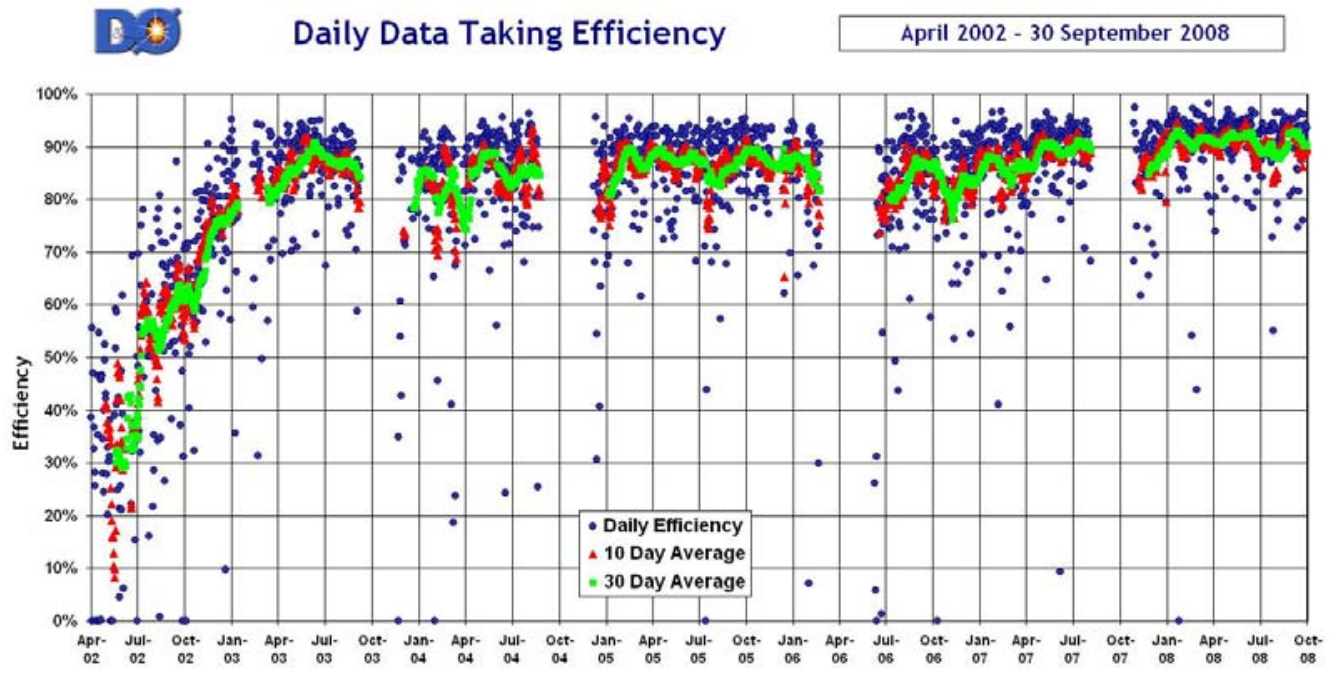

Figure 4. DZero data-taking efficiency as a function of time during Run II. The blue dots represent a daily average, the red triangles represent a 10-day average, and the green squares represent a 30-day average.

DZero used the 2007 shutdown access time to perform maintenance and introduce improvements. The luminosity monitors were replaced due to radiation damage to the scintillators. A substantial effort was undertaken to study, and where possible recover, failed Silicon Microstrip Tracker readout channels. Firmware and software were developed and implemented to utilize a readout feature of the AFEII boards which measures the time of arrival of the light generated in the Central Fiber Tracker. These timing measurements are planned to be used to enhance track purity, and may also be used to suppress accidental hits during track reconstruction. This timing data has been read out since the end of the 2007 shutdown. Also during the shutdown, the readout for the Forward Preshower Detector was instrumented with AFEII boards. The boards enhance reliability while providing the capability to increase the dynamic range of the readout, as well as improve the uniformity of gains. Liquid argon levels in the calorimeters can slowly decrease due to pressure differences between the cryostats and the storage dewar. Consequently, the liquid argon levels were topped off in the south End-Cap Calorimeter and the Central Calorimeter. Significant effort was invested in enhancing the performance of the Inner Cryostat Detector. The muon systems were calibrated, and improvements were made to the slow communications paths for the Proportional Drift Tube detectors. The control room computer nodes were upgraded. The operating systems for the online cluster were upgraded, as were the fileservers. Safety system tests, single channel repairs, and routine and preventive maintenance were completed to prepare the detector for the long operating period. For example, a small leak which had developed in the toroid coil water cooling system was repaired. The Forward Proton Detectors were decommissioned and removed, since the instantaneous luminosities are no longer expected to routinely get low enough to support effective operation of those detectors. The associated veto counters were also removed. Additional efforts were made to isolate the Collision Hall from the Tevatron tunnel to minimize the movement of the low-beta quadrupoles in response to airflow between the Collision Hall and the tunnels. The Accelerator Division re-aligned the low-beta quadrupoles to improve performance, and adjusted the transverse location of the interaction point at DZero, moving it 
closer to the center of the detector. This change required a re-evaluation of the triggering equations used for the Central Track Trigger.

As illustrated in Figure 4, the start-up of the DZero detector after the 2007 shutdown was remarkably smooth and efficient, representing the best shutdown recovery to date. DZero was recording physics-quality data during the first post-shutdown 36-on-36 store. Special triggers to collect calorimeter calibration data were run in parallel with the standard physics suite, and the calorimeter calibration was verified.

DZero continues efforts to streamline operations in the face of increasing instantaneous luminosity, and is refining its operations in an effort to improve efficiency. The operating voltages of the Silicon Microstrip Tracker were adjusted to maintain optimal performance, and monitoring for the impact of radiation damage continues. As expected, the results of this monitoring indicate that it will not be possible to fully deplete all of the Layer 1 silicon by the end of Run II. Layer 0 was installed during the 2006 shutdown, in part to compensate for this anticipated degradation in performance. The operating parameters of the Forward Preshower Detector were optimized to take full advantage of the newly installed AFEII readout electronics. Firmware was developed, tested, and deployed to limit the temporary acceptance losses induced by radiation damage to front-end readout electronics in the muon Proportional Drift Tube system. In addition to ongoing efforts to address remaining sources of dataflow interruptions, there are continuing efforts to improve monitoring tools, and the guidance, training and documentation provided for the rotating shift crews that operate the detector around the clock.

DZero continued to develop and refine its trigger suite in anticipation of the potential for routine operation with initial luminosities near and above $3 \times 10^{32} \mathrm{~cm}^{-2} \mathrm{~s}^{-1}$. An improved trigger list was introduced in March 2008, which included new electron, muon, and tau selections and incorporated terms that suppress multiple interactions. This trigger list is designed to efficiently handle initial luminosities up to $3.5 \times 10^{32} \mathrm{~cm}^{-2} \mathrm{~s}^{-1}$ without prescaling high-priority high- $\mathrm{p}_{\mathrm{t}}$ physics triggers. As of the end of FY 2008, the record initial luminosity at DZero was $3.12 \times 10^{32} \mathrm{~cm}^{-2} \mathrm{~s}^{-1}$, and the trigger and detector performed well under these conditions.

The average operating efficiency of the detector continues its gentle rise, and is now at $87.1 \%$ for the entire Run II data sample. The integrated luminosity recorded during FY 2008 was $40 \%$ larger than any previous fiscal year. As illustrated in Figure 5, the total recorded integrated luminosity increased by $54 \%$ to $4.41 \mathrm{fb}^{-1}$. The average rate of integrating luminosity significantly exceeded the Tevatron design goal from late April through the end of the fiscal year (see Figure 1). 


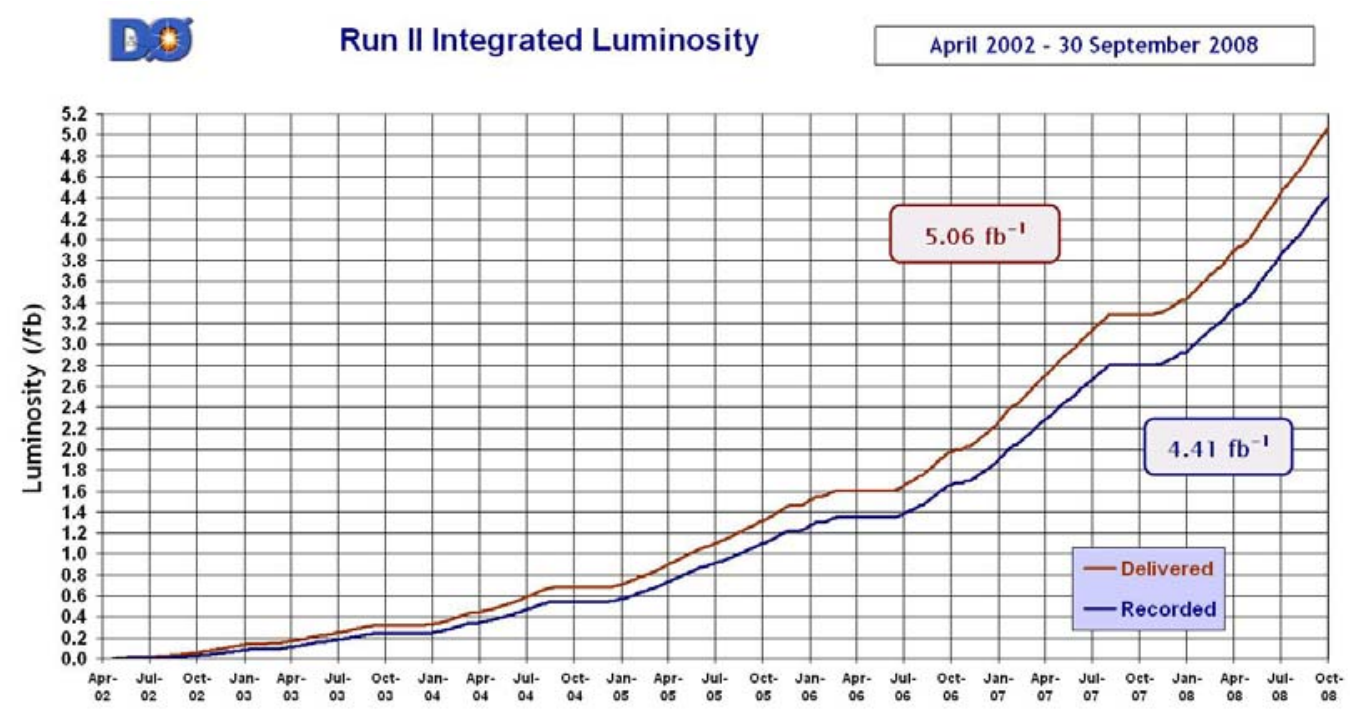

Figure 5. Delivered (red line) and recorded (blue line) luminosity at DZero as a function of time during Run II.

With the significant improvements in antiproton stacking rate achieved by the Fermilab Accelerator Division, the elimination of the long shutdown in 2008, and the ongoing efforts to reduce shot setup time, the prospects for delivery of more than $8 \mathrm{fb}^{-1}$ to each of the collider detectors before the end of FY 2010 look achievable. Also, calendar year 2008 is already a record year for the number of published DZero papers. Given the smooth and efficient operation of the DZero detector, the collaboration is well positioned to take full advantage of increasing luminosity and to continue its multifaceted exploration of the high-energy physics frontier.

NuMI / E-875 Main Injector Neutrino Oscillation Search (MINOS) (A. Habig, J. Hylen, R. Plunkett)

$\underline{\text { NuMI }}$

NuMI was off for the first part of FY 2008 (until November 16, 2007) to finish shutdown activities. Major shutdown work included: installing dehumidifiers in the target/horn cooling air system to aid in tritium mitigation; changing from vacuum to helium in the decay pipe to reduce strain on the pipe's window; and repairing horn 2 by replacing its water suction line. Running for the rest of the year was reasonably smooth. The following downtimes were necessary for repairs: fifteen days to repair horn 2 (replace leaking water supply line); three days to fix magnet QD111 in the proton extraction line, five days to repair a septum in the Booster accelerator; and 40 days to replace horn 1 with a spare horn. The horn 1 failure was a water suction line similar to the horn 2 failure, but the higher residual radioactivity of horn 1 precluded replacement of that line on the horn. Both new horns have suction lines with a different design that should last significantly longer than the old style. Horn 1 had run for more than 3 years and 24 million pulses, well beyond its specified lifetime. The downtime for replacing the horn was extended by the need to remove the target and repair its drive mechanism. 
Several improvements were made to the NuMI beam itself. The most significant improvement was the increase of NuMI beam power by the implementation of slip-stacking in the Main Injector, starting regular operation January 10, 2008. NuMI now runs routinely with 8 Booster turns. Secondly, operations at a 2.2 s cycle time offered a $10 \%$ improvement in power over the previous year's $2.4 \mathrm{~s}$ cycle. These improvements raised the typical mixed-mode performance (coincident NuMI and antiproton production) from $24 \times 10^{12}$ protons/pulse and $170 \mathrm{~kW}$ for NuMI to $30 \times 10^{12}$ protons $/$ pulse and $250 \mathrm{~kW}$. The integrated protons-on-target to NuMI for the year (Figure 6) was $1.99 \times 10^{20}$.

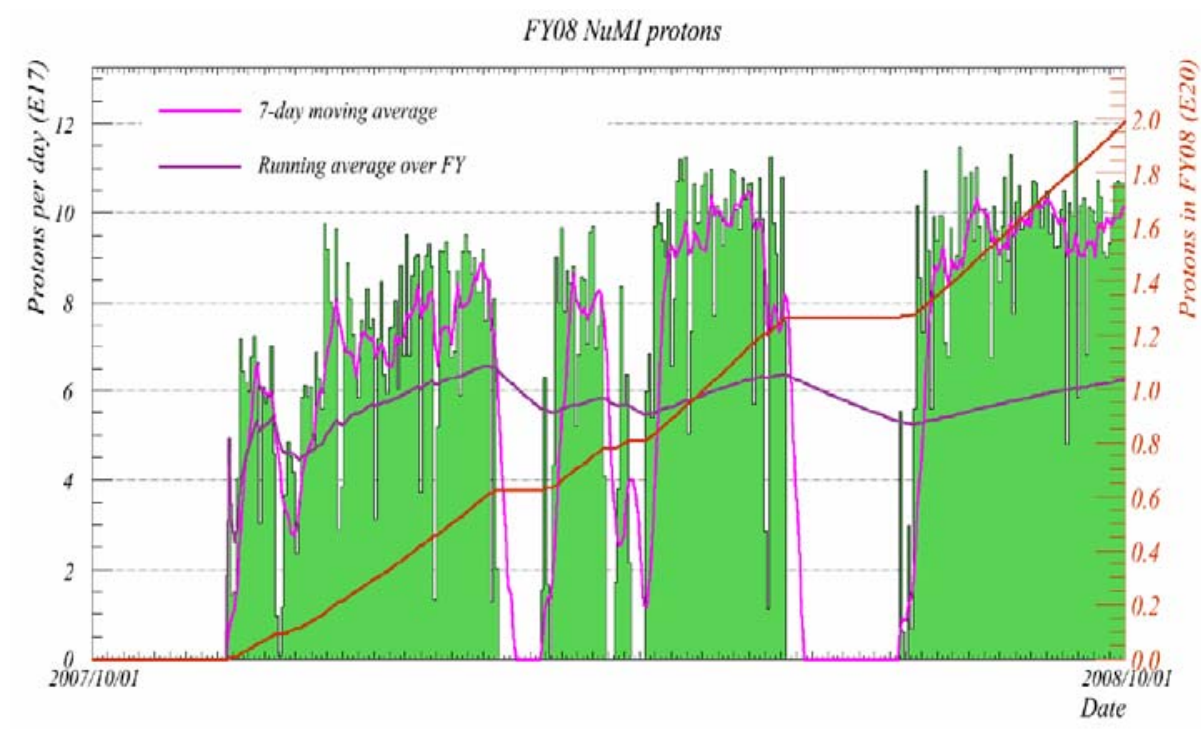

Figure 6. NuMI's daily total protons-on-target (histogram, left axis) and integrated FY 2008 total proton exposure (red line, right axis). A total of $1.99 \times 10^{20}$ protons were delivered to the NuMI target in FY 2008.

\section{$\underline{\text { MINOS }}$}

The MINOS experiment continued efficient operation in FY 2008. Both Near and Far Detectors operated at extremely high efficiency when the beam was on, with the statistics-limited Far Detector taking good data more than $99 \%$ of the time during beam and $98 \%$ of the time overall (including scheduled shutdown maintenance). Typically, the Far Detector has no dead channels, with one problem developing every few weeks and being fixed within a day; so no changes have had to be made there. The Near Detector electronics retrofit reported on last year resulted in a similar problem-free readout, and with the removal of the burden of continuously replacing bad boards, the Near Detector electronics efforts made great strides in solving more subtle problems, resulting in the cleanest data so far. The few down times at the Far Detector were mostly scheduled power outages to replace lightning-damaged power feeds and to install additional lightning arrestors to forestall future such damage. Unscheduled Far Detector downtime for various electronics problems was only a few hours for the whole year. The Near Detector's downtime was dominated by repairs to the analysis-magnet power supply and its reversing switch, and downtime for infrastructure work related to new experiments (MINERvA and ArgoNeuT) moving into the Near Detector Hall. To provide systematic controls on the magnetic-field strength, regular field reversals are now a feature of Near Detector operations, with the field changing sign every two weeks. 
An exposure of $3.36 \times 10^{20}$ protons on target (all the data through the 2007 shutdown) of $v_{\mu}$ data has been used to improve the measurement of $v_{\mu}$ disappearance oscillations, published in FY 2008 [Phys. Rev. Lett. 101, 131802 (2008)]. The increase of data and better analysis techniques have greatly improved our knowledge of the $v_{2} \leftrightarrow v_{3}\left|\Delta \mathrm{m}^{2}\right|$ oscillation parameter, as shown in Figure 7. The oscillation parameters are now measured to be $\Delta \mathrm{m}^{2}=2.43 \pm 0.13 \times 10^{-3} \mathrm{eV}^{2}$ and $\sin ^{2} 2 \theta=1.00_{-0.05}^{+0.00}$ (one sigma errors). The increased precision now disfavors non-standard $\nu_{\mu}$ disappearance models such as neutrino decay (by 3.7 $\sigma$ ) and decoherence (by $5.7 \sigma$ ).

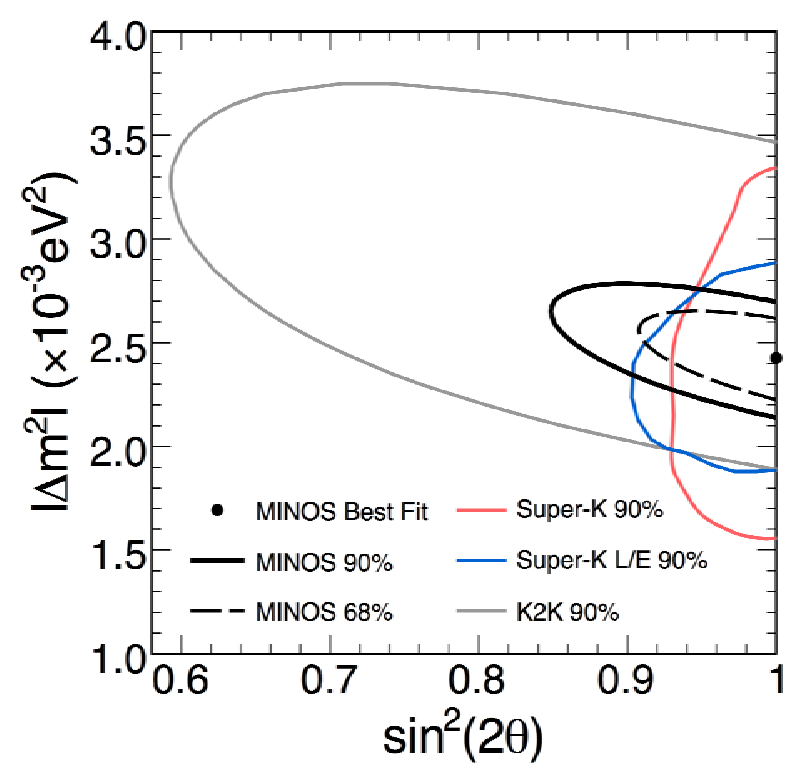

Figure 7. The allowed region in parameter space from the analysis of charged-current $v_{\mu}$ interactions in MINOS resulting from $3.36 \times 10^{20} \mathrm{NuMI}$ protons-on-target, compared to previous experiments.

To check if the observed $v_{\mu}$ disappearance might involve a fourth, sterile neutrino flavor, the rate of Neutral Current (NC) interactions can be compared between the Near and Far Detectors. Such a sterile neutrino would undergo no NC interactions while any of the three known flavors would. An exposure of $2.46 \times 10^{20}$ protons-on-target has been used to make such a comparison, with a Far Detector spectrum compared to expectations derived from the observed high-statistics Near Detector data shown in Figure 8. The data are consistent with no sterile neutrino participation, with a measurement of the fraction of sterile participation $\mathrm{f}_{\mathrm{s}}<0.68$ at $90 \%$ confidence level. The result has been accepted for publication by Physical Review Letters (arXiv:hep-ex/0807.2424). 


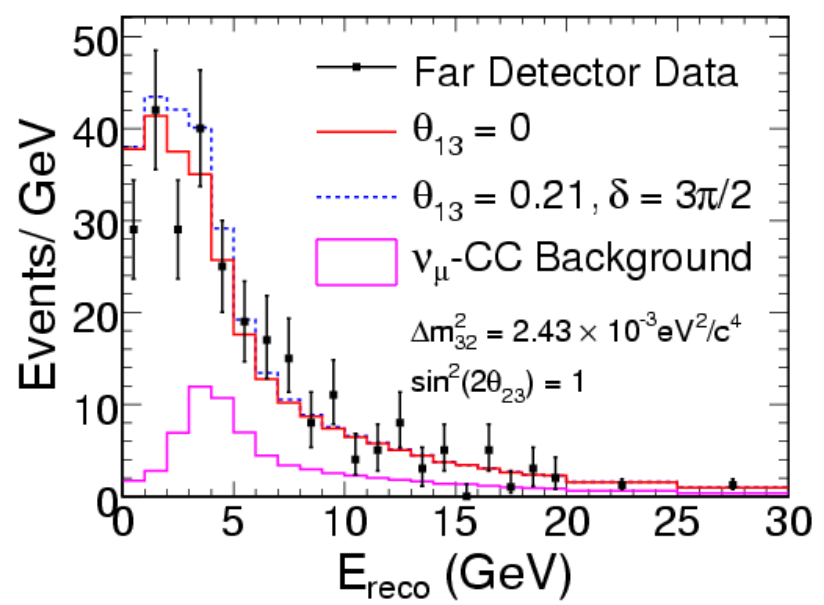

Figure 8. The Neutral-Current-selected event energy-spectrum in the Far Detector. The black dots are the data, the red and blue lines the expectations from the measured Near Detector spectrum under two different $\theta_{13}$ scenarios, and the magenta line is the Charged-Current background.

Other MINOS papers published in FY 2008 include "The Magnetized Steel and Scintillator Calorimeters of the MINOS Experiment" [Nucl. Instr. and Meth. A596, 190-228 (2008)], "Testing Lorentz Invariance and CPT Conservation with NuMI Neutrinos in the MINOS Near Detector" [Phys. Rev. Lett. 101, 151601 (2008)], and "Study of Muon Neutrino Disappearance Using the Fermilab Main Injector Neutrino Beam" [Phys. Rev. D 77, 072002 (2008)].

E-944 / MiniBooNE (S. Brice, R. Van de Water)

FY 2008 Booster Neutrino Beam running for MiniBooNE was in both neutrino and antineutrino modes. The first half of FY 2008 was spent running in neutrino mode as dictated by SciBooNE requirements. MiniBooNE collected a further $1.0 \times 10^{20}$ protons on target for neutrino data. This added an extra $20 \%$ more data to the recent re-analysis of the low-energy excess than reported in the original neutrino oscillation publication. Thus, for neutrino mode, MiniBooNE has a total of $6.5 \times 10^{20}$ protons on target for analysis.

After the end of the neutrino run, starting in May 2008, the Booster Neutrino Beam switched back to antineutrino mode. The goal is $5 \times 10^{20}$ protons on target in antineutrino mode. Around June 2008 NuMI went off for seven weeks. During this time MiniBooNE gained a factor of three increase in beam rate, which resulted in about $\sim 1 \times 10^{19}$ protons on target collected per week. With this exceptional running available, MiniBooNE was able to add a significant amount of data to the antineutrino totals. On October 22, 2008 MiniBooNE opened the antineutrino oscillation box with a total of $3.4 \times 10^{20}$ protons on target, with $1.1 \times 10^{20}$ protons on target coming from the last six months of running. The physics results obtained will be made public December 11, 2008. 
Figure 9 shows the weekly performance of the Booster Neutrino Beam since the start of running in 2002. The antineutrino running is shown in the shaded regions The performance of the Booster in FY 2008 has been exceptional. During the last half of FY 2008, the weekly protons-on-target delivered to the Booster Neutrino Beam was over $4 \times 10^{19}$. What makes this number impressive is that this was while full beam, with slip-stacking implemented, was simultaneously delivered to the NuMI target.

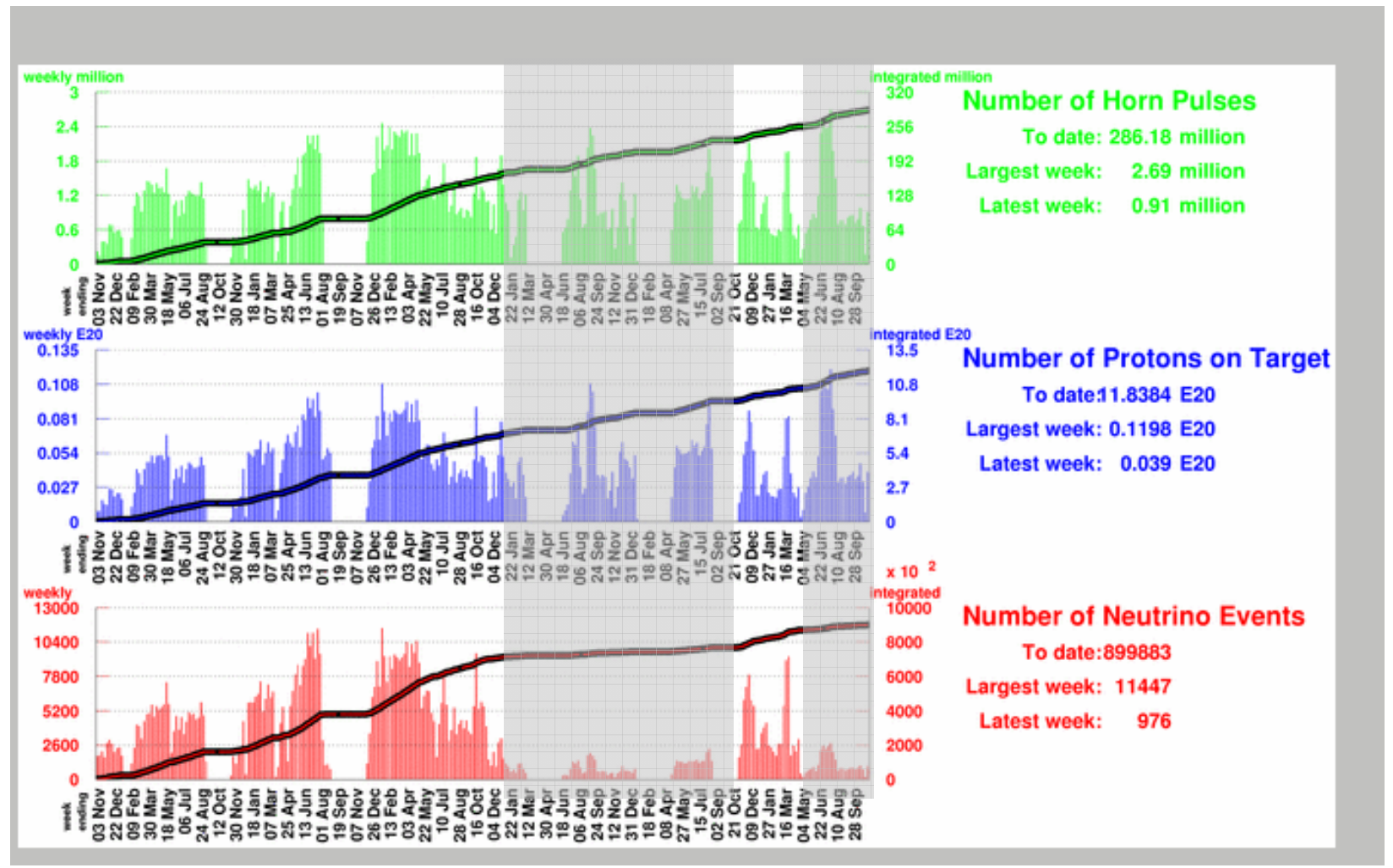

Figure 9. Weekly beam summary since the start of MiniBooNE operation in 2002. Antineutrino running is shown in the two grey-shaded regions.

Figure 10 shows the rate of neutrinos per protons-on-target for antineutrino running. MiniBooNE had the period of running where one, then two, $25 \mathrm{~m}$ absorber plates had fallen into the beam line. After the repairs to the absorber supports, the neutrino event rate went back to normal and remained that way. Subsequent analysis of the data with the absorber problem showed it to be completely recoverable. Detailed modeling of the absorber effects are understood, and the data can be corrected for use in neutrino oscillation and cross section analyses. This absorber data was included in the recent antineutrino box opening data set. 


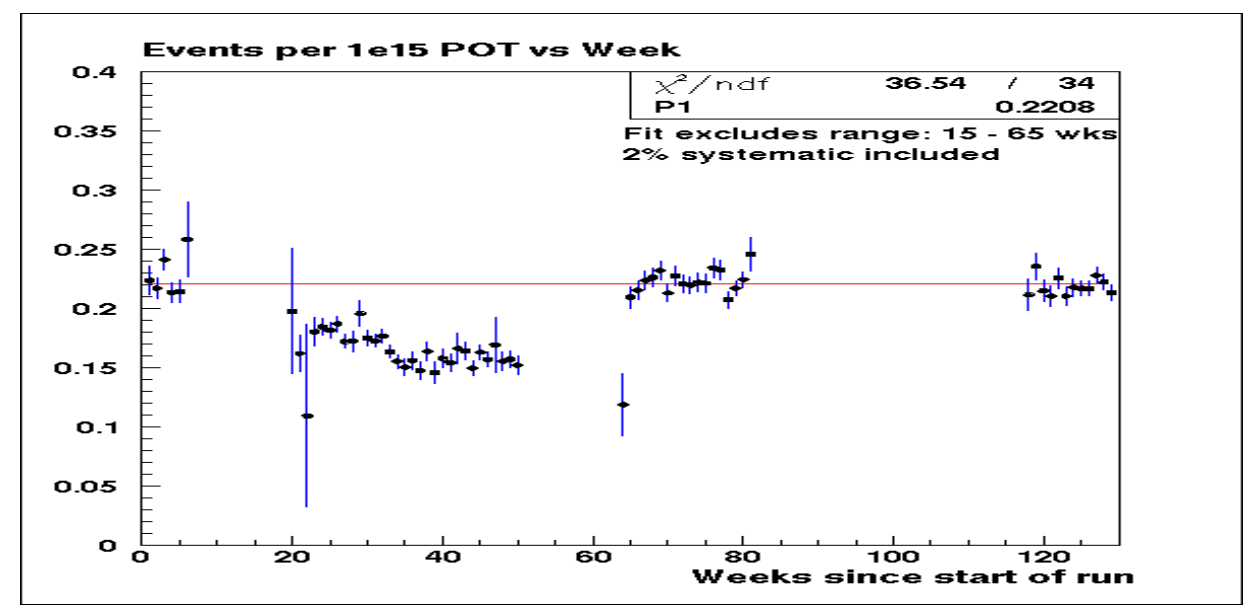

Figure 10. Neutrino events per protons-on-target (POT) for the antineutrino run starting in January 2006. The red line shows the expected value for normal running. After the 2006 long shutdown (first gap), the rates decreased due to the $25 \mathrm{~m}$ absorber problem. A repair was done from week 52 to 64 . Since then, the neutrino event rate has returned to normal, including the latest antineutrino period in FY 2008.

An important aspect of continued running of MiniBooNE is the reliability of the beamline, horn, and detector systems. For the FY 2008 antineutrino run period, the combined beamline and detector uptime was over $95 \%$. The most crucial element, the horn, has collected over 200 million pulses, surpassing the first horn, which failed two years ago with 94 million pulses (both these numbers are world records). This second horn continues to run flawlessly, with no sign of problems. In the unfortunate event of failure, a spare horn and target are available. It would require three weeks to swap in the replacement system.

\section{E-954 / SciBooNE (T. Nakaya, M. Wascko)}

SciBooNE began its first data-taking with a dominantly antineutrino flux. During the 2007 accelerator shutdown, the Booster Neutrino Beam horn-polarity was changed, putting the beam configuration into neutrino mode. Also, detector repairs were performed, so that all detector channels were operational on beam triggers. The SciBooNE neutrino run started on October 13, 2007, and lasted until April 9, 2008, when another horn polarity changeover was begun, returning the beam to antineutrino running. During the neutrino run, data from $1.04 \times 10^{20}$ protons-on-target were collected in total, with data from $0.99 \times 10^{20}$ passing all quality cuts.

The last horn polarity change was completed quickly, and the second period of antineutrino running began on April 14, and lasted until August 18. During the second antineutrino run, data from $1.06 \times 10^{20}$ protons-on-target were collected in total, with $1.01 \times 10^{20}$ protons-on-target passing all quality cuts. The beamline and neutrino horn performed flawlessly over the entire run. In total, SciBooNE collected $2.52 \times 10^{20}$ protons-on-target, which is $26 \%$ more than was requested in the proposal.

Table 3 summarizes all data collected during the SciBooNE data runs. Figure 11 shows the accumulation of protons-on-target per week throughout the run. In the figure, the solid curve 
shows the total protons-on-target, and the dashed curve shows the protons-on-target for events that pass all data quality cuts. The average detector efficiency over the entire run was over $95 \%$. Figure 12 shows the number of charged-current candidate neutrino and antineutrino events normalized by protons-on-target over the duration of the SciBooNE data run. In the figure, the differing event rates in neutrino and antineutrino modes is apparent.

Table 3. SciBooNE beam data collection history.

\begin{tabular}{|l|l|c|}
\hline Run & Dates & Protons-on-target \\
\hline 1 (Antineutrino) & Jun 2007 - Aug 2007 & $0.52 \times 10^{20}$ \\
\hline 2 (Neutrino) & Oct 2007 - Apr 2008 & $0.99 \times 10^{20}$ \\
\hline 3 (Antineutrino) & Apr 2008 - Aug 2008 & $1.01 \times 10^{20}$ \\
\hline
\end{tabular}

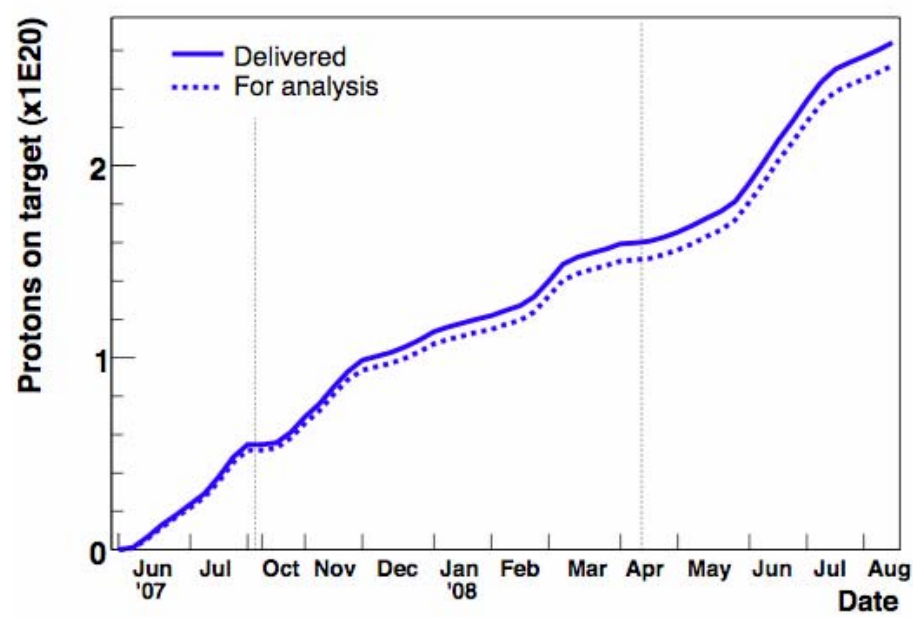

Figure 11. SciBooNE accumulated protons-on-target.

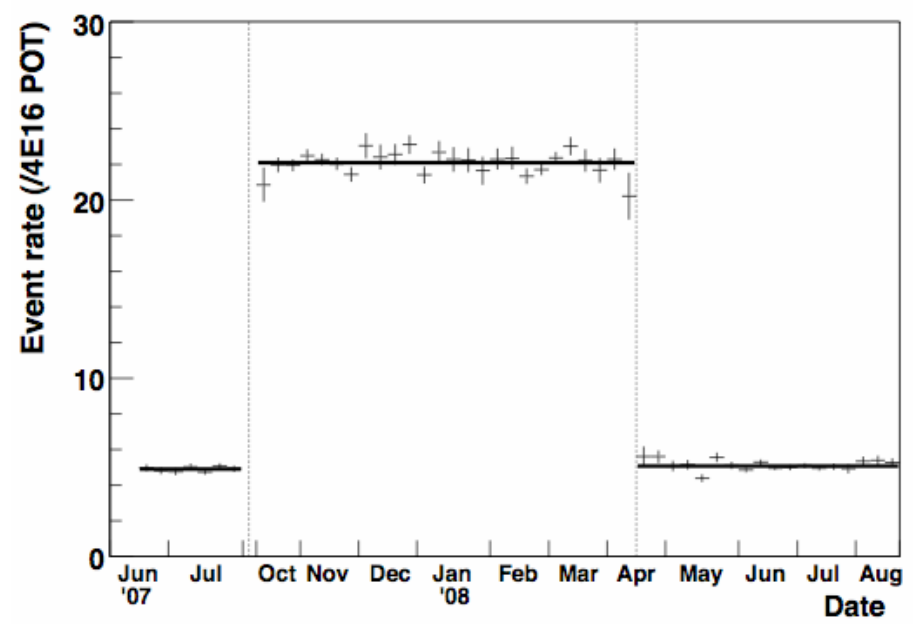

Figure 12. SciBooNE events per protons-on-target. The error bars shown come from data statistics only. 
On August 18 SciBooNE detector decommissioning began. The SciBar and Muon Range Detector elements were decommissioned in parallel, and work was completed by August 29. The Electron Catcher subdetector decommissioning began September 22 and was completed September 29.

SciBooNE was honored by the DOE for its recycling and reuse of detector components. In January 2008, SciBooNE was awarded the Office of Science's Noteworthy Practice award, and in April the experiment was awarded the DOE's Pollution Prevention (P2 Star) award.

Fixed-Target Switchyard 120 GeV (SY120) and MTest (R. Coleman, W. Kissel, E. Ramberg) Introduction to MI Beam Extraction for SY120

The test beam originates from the resonant extraction of at least one Booster batch inside the Main Injector (MI). This batch usually consists of 60-80 RF 'buckets', with buckets separated by $19 \mathrm{nsec}$. Thus the batch is about 1.5 microseconds long. The time required for a particle to transit the full circumference of the MI is about 10 microseconds. The batch or batches are accelerated to $120 \mathrm{GeV}$, circulate around the MI, and are slowly extracted over a macroscopic slow spill. For most usage of the test beam, there will be less than $100 \mathrm{kHz}$ of beam. If beam were smoothly extracted, this would imply that, at most, one particle per rotation of the MI batch will occur. The beam is not that smooth, and there is up to $35 \%$ double occupancy per MI rotation.

The length and duty cycle of the spill is determined by the Accelerator Division (AD), with guidance from the Program Planning Office. For FY 2008 there was a single 4-second-long spill per minute, for a maximum of up to 14 hours per day. The AD has set up a procedure for easily changing from this four-second spill to a one-second spill. This shorter spill could then be delivered more frequently for commissioning purposes, and for those groups which are limited by data-acquisition buffers. The Accelerator Division also started commissioning a "pinged" beam operation with up to 4 pings per spill with a width for each ping set to 1 to $5 \mathrm{~ms}$.

The beam has an approximately $0.3 \%$ momentum spread and can be focused to a $7 \mathrm{~mm}$ rms spot size for $120 \mathrm{GeV}$ protons, and 1-10\% momentum spread with approximately $2-5 \mathrm{~cm}$ rms spot size for secondary beams of lower momenta.

\section{$\underline{\text { SY120 in FY } 2008}$}

The number of SY120 pulses with beam in FY 2008 was 82,455. During FY 2007, time was taken to rebuild the MTest beamline to optimize the low-energy particle production. ILC detector groups were particularly interested in low-energy pion beams capable of going as low as $1 \mathrm{GeV}$. During FY 2008, several user groups ultilized this new low-energy capability. In addition to the ability to run at much lower energy, the new beamline had several other improvements in instrumentation and tuning facilities which improved general operations. This new beamline resulted in a vastly superior ability to deliver low-energy beam to the user areas. The resulting beam rates are given in Table 4 . 
Table 4. Rate of beam delivered to the MT6 user facility for $1 \times 10^{11}$ protons in the Main Injector. Identification of electrons is by lead glass calorimetry.

\begin{tabular}{|c|c|c|c|c|}
\hline $\begin{array}{c}\text { Beam Energy } \\
(\mathrm{GeV})\end{array}$ & $\begin{array}{c}\text { Rate at Entrance to } \\
\text { MT6 (per spill) }\end{array}$ & $\begin{array}{c}\text { Rate at Exit of } \\
\text { MT6 (per spill) }\end{array}$ & $\begin{array}{c}\text { \%Pions, Muons } \\
\text { at exit of MT6 }\end{array}$ & $\begin{array}{c}\text { \% Electrons at } \\
\text { exit of MT6 }\end{array}$ \\
\hline 16 & 132,000 & 95,000 & $82 \%$ & $18 \%$ \\
\hline 8 & 89,000 & 65,000 & $42 \%$ & $58 \%$ \\
\hline 4 & 56,000 & 31,000 & $26 \%$ & $72 \%$ \\
\hline 2 & 68,000 & 28,000 & $34 \%$ & $65 \%$ \\
\hline 1 & 69,000 & 21,000 & $<50 \%$ & $>50 \%$ \\
\hline
\end{tabular}

The rates measured in the new beamline matched expectations, and the new secondary beam is much easier to operate. The beamline was set to the following momenta for various users: $120 \mathrm{GeV} / \mathrm{c}, 66 \mathrm{GeV} / \mathrm{c}, 32 \mathrm{GeV} / \mathrm{c}, 16 \mathrm{GeV} / \mathrm{c}, 8 \mathrm{GeV} / \mathrm{c}, 4 \mathrm{GeV} / \mathrm{c}, 2 \mathrm{GeV} / \mathrm{c}$ and $1 \mathrm{GeV} / \mathrm{c}$. The primary proton intensity was changed by varying the number of Main Injector turns and Booster bunches (from 1 turn, 5 bunches to 2 turns, 84 bunches).

\section{The User Facility}

The test beam user facility gives users from around the world an opportunity to test the performance of their particle detectors in a variety of particle beams. A plan view of the facility is shown in Figure 13. The web site for the MTest facility can be found at http://www-ppd.fnal.gov/MTBF-w/.

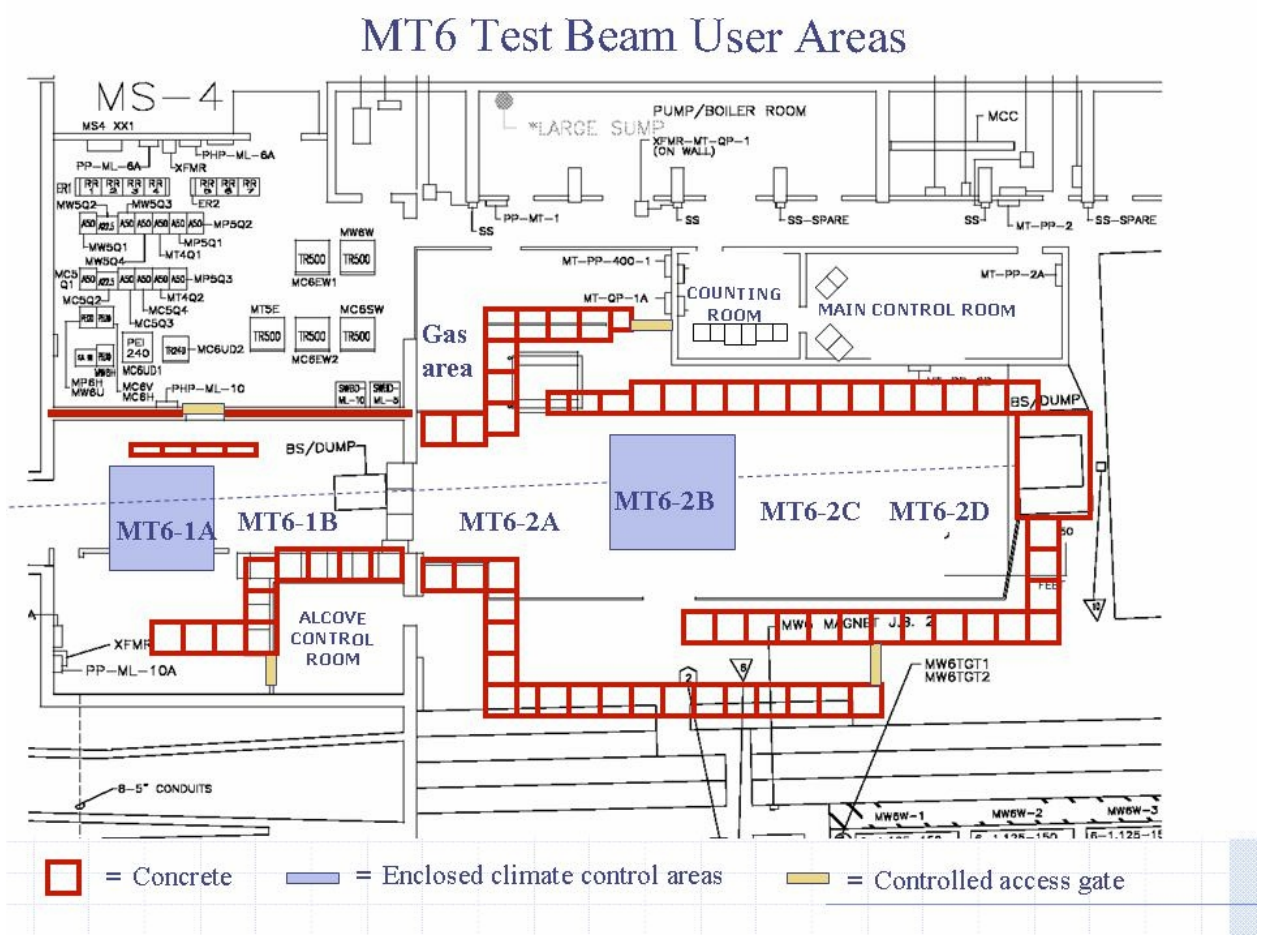

Figure 13. Plan view of the Meson Test Beam Facility. 


\section{Research performed at the MTest Facility}

Each test beam experiment is required to prepare a Memorandum of Understanding with the Laboratory, in which beam, infrastructure, and safety requirements are spelled out in detail. Six new experiments were approved and took data during FY 2008. An additional experiment that took data in FY 2007 returned to take additional data in FY 2008. These seven experiments are listed in Table 5. These experiments took data for a total of 18 weeks. In addition, there were two weeks devoted to major installations of new equipment and two weeks devoted to beam studies for the facility.

Table 5. MTest experiments performed in FY 2008.

\begin{tabular}{|l|l|}
\hline Test & Description \\
\hline & \\
\hline T-984 & PHENIX Vertex Detectors \\
\hline T-979 & Ultra Fast Timing \\
\hline T-978 & CALICE - ILC Calorimetry \\
\hline T-977 & MINERvA Experiment \\
\hline T-976 & CsI Timing Experiment \\
\hline T-971 & LHCb Silicon Detector Upgrade \\
\hline & \\
\hline T-953 & U. Iowa Cerenkov Light Tests \\
\hline
\end{tabular}

Two of these experiments, CALICE and MINERvA, involved very significant installations inside the user area.

The CALICE experiment (T-978) is a collaboration among a large number of mostly European groups, headed in large part by DESY, that is devoted to studying advanced techniques of calorimetry for the ILC. The installation of the CALICE apparatus in the test beam in April, 2008 was substantial, involving veto detectors, drift chambers, electromagnetic calorimetry, hadronic calorimetry, and muon identification. A photo of the final setup is shown in Figure 14. The finely granulated hadronic calorimeter was useful in identifying beam species. Figure 15 shows the measurements of relative flux of particle species, which matches reasonably well the numbers calculated in Table 4 using cruder techniques.

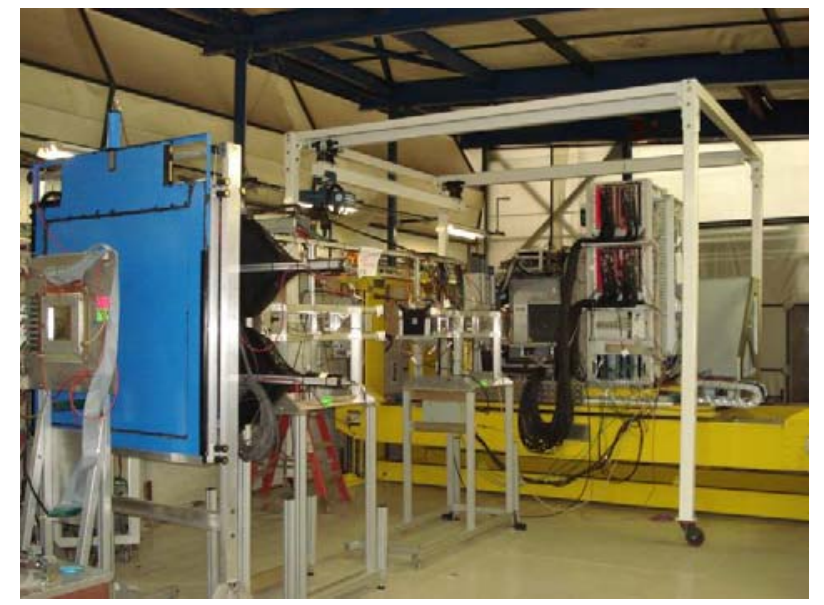

Figure 14. The CALICE experiment setup in the MTest Facility. 


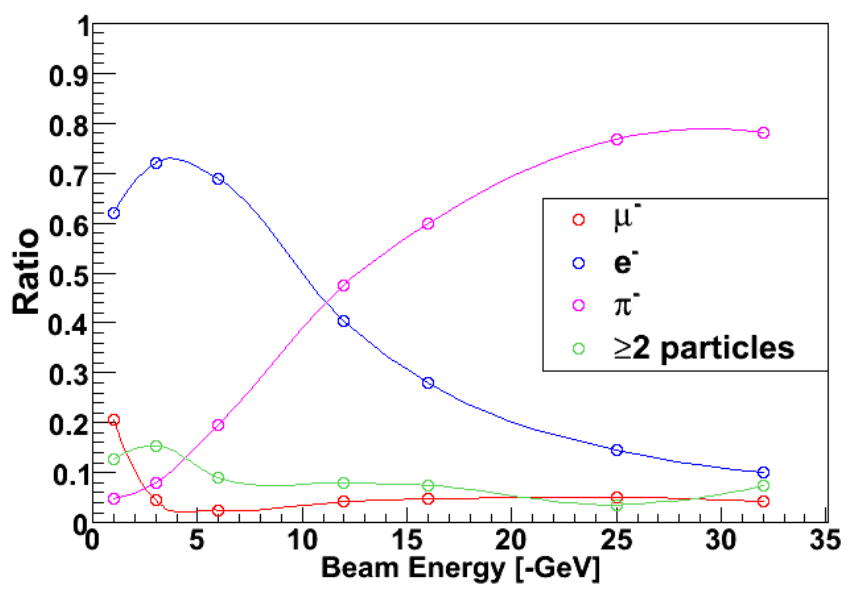

Figure 15. Beam composition as determined by CALICE.

The MINERvA experiment (T-977) proposed installing an entirely new tertiary beamline in the user facility to deliver $300 \mathrm{MeV} / \mathrm{c}$ pions onto the test apparatus. The installation of this beamline was begun in FY 2008 and will be completed in the first part of calendar year 2009. A schematic drawing of the new beamline is shown in Figure 16. After completion of the MINERvA tests, this beamline will be available for other users. The target and collimater can be rolled quickly aside so that the facility can also operate as before.

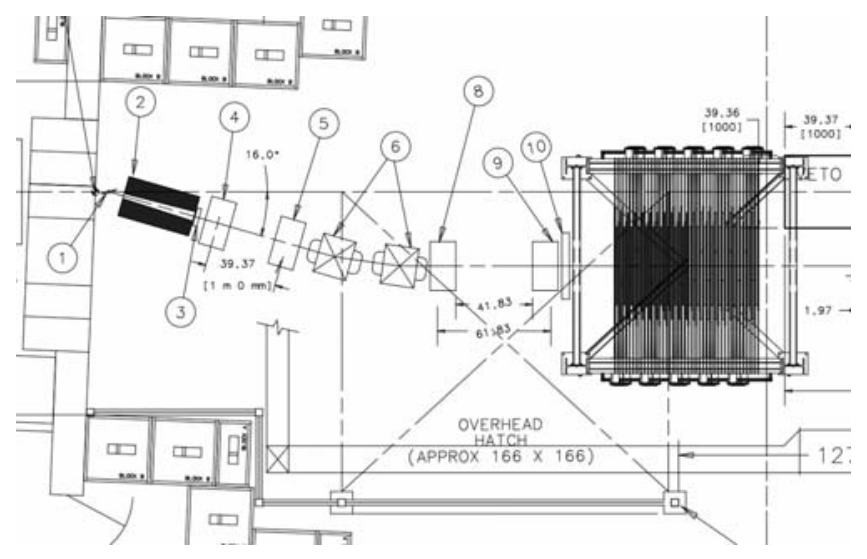

Figure 16. Schematic of the MINERvA tertiary beamline installation.

Experiment T-979 was a test of ultra-fast timing properties of microchannel plate photomultiplier tubes (MCP/PMT). With the help of grant money from the Strategic Collaborative Initiative with the University of Chicago, equipment was purchased for use in this test, which successfully achieved better than $15 \mathrm{psec}$ resolution for time-of-flight measurements. These MCP/PMT units will become an operational part of the facility, thus improving its particle identification capability.

These three experiments are examples of how Fermilab works closely with visiting teams. Not only does Fermilab deliver high-quality beams, but the Test Beam Facility may retain experiment-provided beam monitoring equipment for future use. 